\title{
Impact of targeted attack on the spontaneous activity in spatial and biologically-inspired neuronal networks
}

Cite as: Chaos 29, 083126 (2019); https://doi.org/10.1063/1.5099038

Submitted: 05 April 2019 . Accepted: 08 August 2019 . Published Online: 28 August 2019

Sergio Faci-Lázaro (D), Jordi Soriano (D), and Jesús Gómez-Gardeñes (iD)

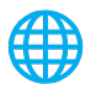

\section{AIP Author Services} English Language Editing 


\title{
Impact of targeted attack on the spontaneous activity in spatial and biologically-inspired neuronal networks
}

\author{
Cite as: Chaos 29, 083126 (2019); doi: 10.1063/1.5099038 \\ Submitted: 5 April 2019 . Accepted: 8 August 2019 . \\ Published Online: 28 August 2019
}

Sergio Faci-Lázaro, 1,2 (D) Jordi Soriano, 3,4 (iD and Jesús Gómez-Gardeñes ${ }^{1,2}$ (D)

\begin{abstract}
AFFILIATIONS
${ }^{1}$ GOTHAM Lab, Institute for Biocomputation and Physics of Complex Systems (BIFI), University of Zaragoza, 50018 Zaragoza, Spain

${ }^{2}$ Department of Physics of Condensed Matter, University of Zaragoza, 50009 Zaragoza, Spain

${ }^{3}$ Departament de Física de la Matèria Condensada, Universitat de Barcelona, E-08028 Barcelona, Spain

${ }^{4}$ Institute of Complex Systems (UBICS), Universitat de Barcelona, E-08028 Barcelona, Spain
\end{abstract}

Note: This paper is part of the Focus Issue on Symmetry and Optimization in the Synchronization and Collective Behavior of Complex Systems.

\begin{abstract}
We study the structural and dynamical consequences of damage in spatial neuronal networks. Inspired by real in vitro networks, we construct directed networks embedded in a two-dimensional space and follow biological rules for designing the wiring of the system. As a result, synthetic cultures display strong metric correlations similar to those observed in real experiments. In its turn, neuronal dynamics is incorporated through the Izhikevich model adopting the parameters derived from observation in real cultures. We consider two scenarios for damage, targeted attacks on those neurons with the highest out-degree and random failures. By analyzing the evolution of both the giant connected component and the dynamical patterns of the neurons as nodes are removed, we observe that network activity halts for a removal of $50 \%$ of the nodes in targeted attacks, much lower than the $70 \%$ node removal required in the case of random failures. Notably, the decrease of neuronal activity is not gradual. Both damage scenarios portray "boosts" of activity just before full silencing that are not present in equivalent random (Erdös-Rényi) graphs. These boosts correspond to small, spatially compact subnetworks that are able to maintain high levels of activity. Since these subnetworks are absent in the equivalent random graphs, we hypothesize that metric correlations facilitate the existence of local circuits sufficiently integrated to maintain activity, shaping an intrinsic mechanism for resilience.
\end{abstract}

Published under license by AIP Publishing. https://doi.org/10.1063/1.5099038

Understanding the robustness of brain circuitry is of utmost importance for tackling degenerative diseases such as Parkinson's and Alzheimer's. One avenue to address this challenge is the study of neuronal cultures, as they are systems whose structure and dynamics can be controlled and monitored in real time, thus providing useful insights into the collective functioning of ensembles of brain neurons. Here, by taking advantage of the current knowledge about the structural and dynamical patterns of real neuronal cultures, we construct synthetic networks displaying similar wiring patterns and study their behavior under node removal, mimicking the damage experienced by neuronal circuits under degenerative diseases. We compare the effects of targeted attacks and random failures by monitoring the evolution of the structural and dynamical patterns as neurons are sequentially removed. In both cases, activity crisis occurs well before the structural disintegration of the network. In addition, our numerical results illustrate that activity changes occur sharply, pointing out that, although the network can maintain activity for a large fraction of neurons removed, at the vicinity of critical regions a tiny fraction of neuron removal triggers network silence. Interestingly, we observe that metric correlations facilitate neuronal activity to be retained in small localized circuits before the whole activity ceases. This result pinpoints a mechanism that may be key for explaining the resilience to damage of neuronal cultures.

\section{INTRODUCTION}

A fundamental aspect in the understanding of brain damage is the impact of node centrality and network topology on the 
circuit structural integrity and activity flow. ${ }^{1-3}$ It was observed that Alzheimer's and Parkinson's cause a significant loss of hubs and deteriorate the small-world organization of the neuronal circuits, altering synchronization patterns or the integration-segregation balance. ${ }^{4-6}$

Given the complexity of brain circuits, and the inherent difficulty to explore damage in a controlled manner, experimental investigations have been combined in recent years with in silico numerical models. ${ }^{3,7}$ The goal is to better comprehend the impact of damage on the network structure, shed light on the importance of node centrality, and make predictions on the dynamical alterations at whole-network scale. These models have also underscored the importance to distinguish between "targeted attack," i.e., the deletion of central nodes, and "failure," i.e., the deletion of arbitrary nodes. ${ }^{9}$ For instance, Joyce et al. ${ }^{8}$ applied these two damaging actions to functional brain networks, explored the behavior of the networks and their dynamics as damage progressed, and exposed resilience features.

Despite these advances at the brain scale, there is a substantial interest in the development of numerical models that approach damage at a microcircuit level. ${ }^{10-12}$ The advantage of such models is that they can incorporate diverse connectivity distributions and detailed neuronal dynamics while accessing the entire neuronal population. Then, the changes in network collective behavior can be explored according to different damage schemes, to finally unveil the capacity of the system to maintain fundamental traits such as network integrability ${ }^{10}$ or large-scale synchronization. ${ }^{1}$

However, an aspect that is often disregarded when modeling neuronal microcircuits is the inherent spatial embedding of neurons and connections in biological neuronal networks. The brain combines both two-dimensional and three-dimensional organization; and neuronal cultures, ${ }^{13}$ one of the most celebrated experimental model systems in neuroscience, are typically prepared by growing neurons and connections on a flat two-dimensional substrate. ${ }^{14-16}$ Recent studies showed that spatial embedding in these systems naturally feature metric correlations that shape in great measure the architectural and dynamic traits of the neuronal network. ${ }^{14,16-18}$

To the best of our knowledge, there exist no computational studies for damage that take into account the spatial embedding of biologically-realistic neuronal microcircuits. Thus, in the present study, we aim at filling this gap by introducing a detailed numerical exploration of the differences between targeted attack and failure in spatially-embedded networks. Our model is based on the construction introduced by Orlandi et al. ${ }^{14}$ which shapes a spontaneously active network. As major results, we show that the output degree is the most important nodal property for targeted attack, and that much less nodes are required to silence a network in a targeted attack as compared to failure. Apart from these differences, we also show that in both cases, the fall of activity is not gradual but is characterized by sudden boosts that are not present in random (Erdös-Rényi, ER) graphs with identical features but no metric correlations. Our study pinpoints the importance of spatial embedding in the analysis of damage in neuronal networks and aims at providing a starting point to model resilience to insult in neuronal cultures.

\section{THE MODEL}

In this study, we consider a two-dimensional network with $N=500$ nodes (neurons) uniformly spread over a surface of $2 \times 2 \mathrm{~mm}^{2}$. The structure of the network is grounded on a biological construction governed by axonal length, size of the dendritic tree, and neuron-to-neuron connectivity probability. In its turn, the dynamics of the network is portrayed according to an Izhikevich model that incorporates biological noise terms to facilitate spontaneous activity. In Secs. II A and II B, we describe in detail both the structural and dynamical rules governing our synthetic neuronal cultures.

\section{A. Structural model}

According to Graph Theory, complex networks can be defined as a set of nodes $V=\{1,2, \ldots, N\}$ that represent the elements of our system, and a set of edges $L$ that describe the interactions between these elements. ${ }^{19}$ In our case, nodes are excitatory neurons and links are synaptic connections. Network structure is represented through a binary adjacency matrix $A$, with $A_{i j}=1$ for the existence of a connection between neurons $i$ and $j$, and $A_{i j}=0$ otherwise.

Network construction follows biological principles derived from the analysis of connectivity and dynamics in two-dimensional neuronal cultures. Our construction follows the same steps as Orlandi et al. ${ }^{14}$ as follows:

(i) $N$ neurons are spread in a square surface set with periodic boundary conditions to reduce finite size effects. Neuronal spatial arrangement is uniform with density $\rho=125$ neurons $/ \mathrm{mm}^{2}$.

(ii) Each neuronal cell body (soma) is described as a circular object with fixed diameter $\phi_{s}=15 \mu \mathrm{m}$, and its dendritic tree is represented as a concentric circular area with diameter $\phi_{d}$ [Fig. 1(a)]. Dendritic sizes in the network are Gaussian distributed with mean $\mu_{d}=300 \mu \mathrm{m}$ and standard deviation $\sigma_{d}^{2}=40 \mu \mathrm{m}$.

(iii) Axons are incorporated as concatenated segments $\Delta l=10 \mu \mathrm{m}$ long and full length $l$. The distribution of axonal lengths in the network is given by a Rayleigh distribution with average $\langle l\rangle=1.0 \mathrm{~mm}$ and variance $\sigma_{l}^{2}=800 \mu \mathrm{m}$. As shown in Fig. 1(a), the first segment is placed at the end of the soma, and with an orientation that follows a uniform angular distribution. The $i$ th segment is then placed at the end of the previous one and oriented following a Gaussian distribution. To ensure that axons are quasistraight filaments, the standard deviation of the distribution is small, by $\sigma_{\theta}^{2}=0.1 \mathrm{rad}$.

(iv) The connection among two neurons is analyzed after the placement of a new segment. Two neurons may form a synaptic connection when both a geometrical and a statistical conditions are met [Fig. 1(b)]:

- Geometrical condition: the axon of a neuron intersects the dendritic tree of another neuron.

- Statistical condition: the connection is established with probability $\alpha$. This probability is independent of the overlapping length between the axon and the dendritic tree that is intersected.

(v) Valid connections are set in the adjacency matrix, and the process repeated from step (iii) onward until all axons are set and all interneuronal connections explored. 


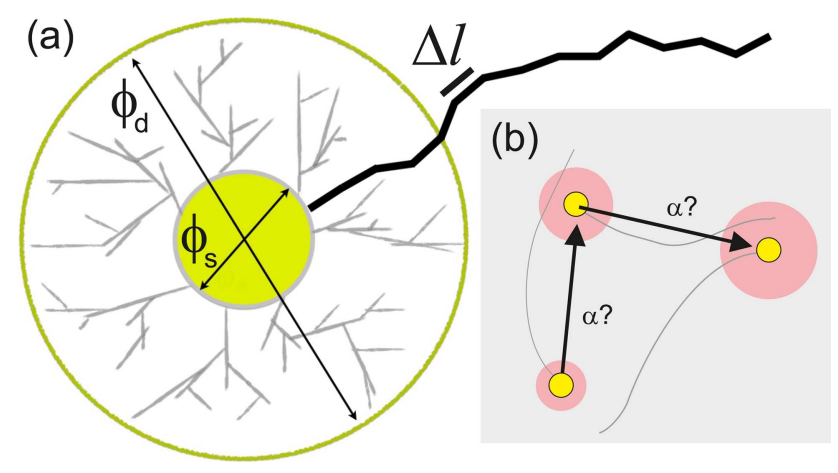

FIG. 1. Representation of a neuron according to our model. $\phi_{s}$ is the diameter of the soma. The average maximum length of the dendrites is the value we take as the radius of the dendritic tree, $\phi_{d} / 2$.

Let us note that, in this construction scheme, the layout of potentially connectable neurons is placed first, shaping the geometrical constrains. Our method is biologically more realistic than those purely statistical models in which connectivity is just distancedependent. ${ }^{2}$

The constructed networks can be characterized by their average connectivity $\langle k\rangle$ and the size of the giant component $G$. As shown in Fig. 2, these two quantities depend on the connectivity probability $\alpha$, The average connectivity $\langle k\rangle$ slowly increases with $\alpha$, in contrast with the giant component $G$ that abruptly grows. This behavior indicates that the constructed networks become globally connected with $\alpha \approx 0.02$, i.e., with just a small fraction of available links. The addition of more links not only strengthens the backbone of the network but also facilitates neuron-to-neuron communication that is central for global network activity (GNA), as shown later.

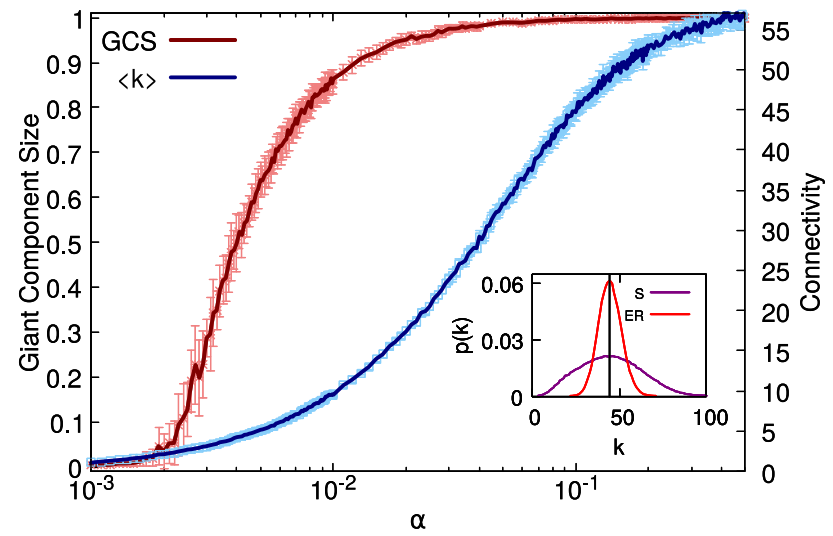

FIG. 2. Dependence of the size of the giant component $G$ and average connectivity $\langle k\rangle$ on the connectivity probability between two neurons $\alpha$. Both quantities increase with $\alpha$, but $G$ grows much faster, reaching a maximum by $\alpha \approx 0.02$. Data are an average on $n=20$ network realizations. The inset shows a comparison between the degree distribution of the spatial synthetic cultures $(\alpha=0.1)$ and that of random ER graphs with similar average connectivity $\langle k\rangle=45$.

\section{B. Dynamical model}

Neuronal dynamics is incorporated through the Izhikevich model ${ }^{21}$ an efficient yet biologically plausible model for a spiking neuron. The model considers the integration of stimuli in the neuronal somas and the transmission of the electrical signal among neurons through their synapses.

\section{Soma dynamics}

Following Refs. 21 and 22, the Izhikevich model is constructed as an integrate-and-fire system with adaptation, and given by two coupled nonlinear ordinary differential equations of the form

$$
\begin{gathered}
\tau_{c} \frac{d v^{j}}{d t}=k\left(v^{j}-v_{r}\right)\left(v^{j}-v_{t}\right)-u^{j}+I^{j}+\eta, \\
\tau_{a} \frac{d u^{j}}{d t}=b\left(v^{j}-v_{r}\right)-u^{j}, \\
\text { if } v^{j} \geqslant v_{p} \longrightarrow\left\{\begin{array}{l}
v^{j} \longleftarrow v_{c}, \\
u^{j} \longleftarrow u^{j}+d .
\end{array}\right.
\end{gathered}
$$

Here, Eq. (1) describes the evolution of the jth soma's membrane potential $v^{j}(t)$, where $\tau_{c}$ is the leaky capacitance, $v_{r}$ is the resting potential, $v_{t}$ is the threshold potentials, $I^{j}$ is the total synaptic current from the rest of the network that stimulates the $j$-neuron, and $\eta$ a noise term. Equation (2) describes the evolution of the jth soma's inhibitory currents $u^{j}(t)$, where $\tau_{a}$ is the main time-scale of the inhibitory current, $b$ is its sensitivity to subthreshold fluctuations of the membrane potential, and $d$ is a parameter that accounts for the adaptation and recovery of the neurons.

The dynamics of the soma is governed by the resting $v_{r}$ and threshold $v_{t}$ potentials of Eq. (1). Since they are, respectively, stable and unstable fixed points, insufficient stimulation maintains the membrane potential below the threshold condition, relaxing toward its resting value $v_{r}$ in the absence of any stimulus. Repeated stimulation brings the membrane potential $v$ over the threshold, which grows rapidly up to its peak value $v_{p}$ and triggering the generation of a spike. The potential is thereafter reset to $v_{c}$.

\section{Synaptic dynamics}

The synaptic dynamics describes the interaction between neurons. The generation of a spike by the $i$ th neuron at time $t_{m}$ induces a postsynaptic current in the $j$ th neuron. Thus, the total synaptic current $I_{j}$ received by the $j$ th neuron reads

$$
\begin{aligned}
I_{j}(t) & =\sum_{i=1}^{N} \sum_{t_{m}<t} A_{i j} E_{i}\left(t, t_{m}\right), \\
E_{i}\left(t, t_{m}\right) & =g_{A} D_{i}\left(t_{m}\right) \exp \left(-\frac{t-t_{m}}{\tau_{A}}\right) \Theta\left(t-t_{m}\right) .
\end{aligned}
$$

Here, $E_{i}\left(t, t_{m}\right)$ is the current induced by the $i$ th neuron at time $t$ as a result of the spikes elicited at time $t_{m}, g_{A}$ is the strength of the synapse, and $\tau_{A}$ is the characteristic decay time of the postsynaptic current. $D_{i}(t)$ is the short-term depression that accounts for the efficiency of 
the neuron presynaptic terminals and is given by ${ }^{22-24}$

$$
\frac{d D_{i}}{d t}=\frac{1-D_{i}}{\tau_{D}}-(1-\beta) D_{i} \delta\left(t-t_{m}\right),
$$

where $\tau_{D}$ is the characteristic recovery time associated to vesicle recycling and $\beta$ is a dimensionless coefficient related to the progressively loss of efficiency of the presynaptic terminals $(\beta<1)$. Consequently, using the initial conditions $D_{i}(t=0)=1$ and assuming that two spikes are generated by the $i$ th neuron at times $t_{m}^{(1)}$ and $t_{m}^{(2)}$, with $t_{m}^{(1)}<t_{m}^{(2)}$, the evolution of $D_{i}(t)$ reads

$$
D(t)= \begin{cases}1-\left[1-D\left(t_{m}^{(1)}\right)\right] \exp \left(-\frac{t-t_{m}^{(1)}}{\tau_{A}}\right) & t_{m}^{(1)}<t<t_{m}^{(2)}, \\ \beta D\left(t \rightarrow t_{m}^{(2)-}\right)=\beta D\left(t_{m}^{(2)}-\delta t\right) & t=t_{m}^{(2)} .\end{cases}
$$

The term $\eta$ of Eq. (1) accounts for noise in the neuronal network. Two sources of noise are considered. The first one is a Gaussian white noise associated to fluctuations in the membrane potential. The second one is a shot noise that accounts for the spontaneous release of neurotransmitters in the presynaptic terminals. These spontaneous releases lead to small currents (termed "minis") in the postsynaptic terminals that are integrated in the soma. Minis are, therefore, analogous to evoked synaptic currents, only differing in their intensity.

Both noise terms are important. They facilitate the spontaneous activation of neurons and the amplification of small signals by the network, and ultimately help orchestrating whole-network coherent activations. $^{14}$

\section{RESULTS}

Once the basic dynamical and structural building blocks of the synthetic cultures are introduced, we now report the main numerical findings, namely, (i) the emergence of spontaneous coherent dynamics (Sec. III A), (ii) the analysis of different attack strategies (Sec. III B), and (iii) the structural and dynamical effects of intentional and random damages (Sec. III C).

\section{A. Emergence of coherent activity}

We start the analysis of our in silico networks by exploring their capacity to exhibit collective behavior, a fundamental trait of all living neuronal circuits. This property will be used later to quantify the impact of damage.

We first explore the dynamical behavior for different connectivity probabilities $\alpha$. Figure 3 depicts representative raster plots for 4 values of $\alpha$, which were selected to cover the typical dynamic scenarios of our simulations, ranging from weak, uncorrelated activity to very strong coherent activity. For $\alpha=0.01$, neurons activate in a sporadic and random manner. Although the giant component almost comprises the entire system, the connectivity is not sufficient to support synchronized dynamics. Rich activity starts to emerge at $\alpha=0.025$, which corresponds to a fully connected network, (a)

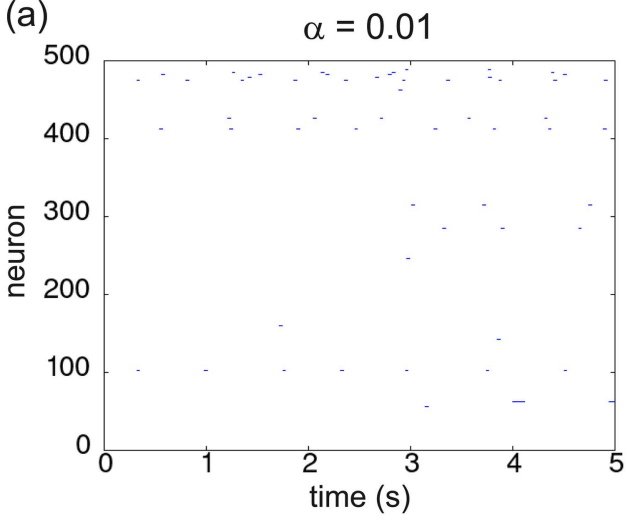

(c)

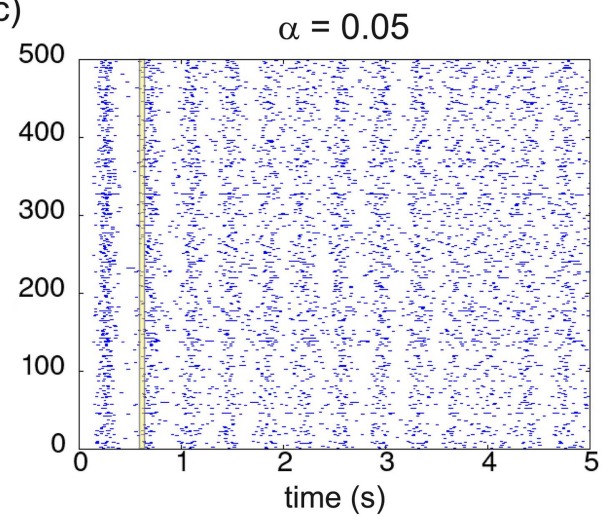

(b)

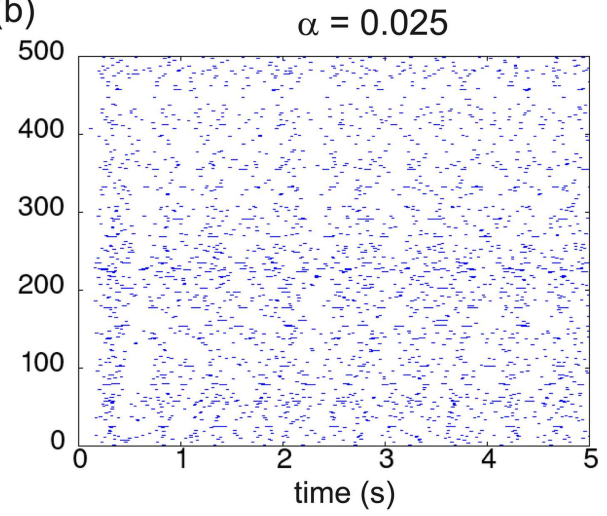

(d)

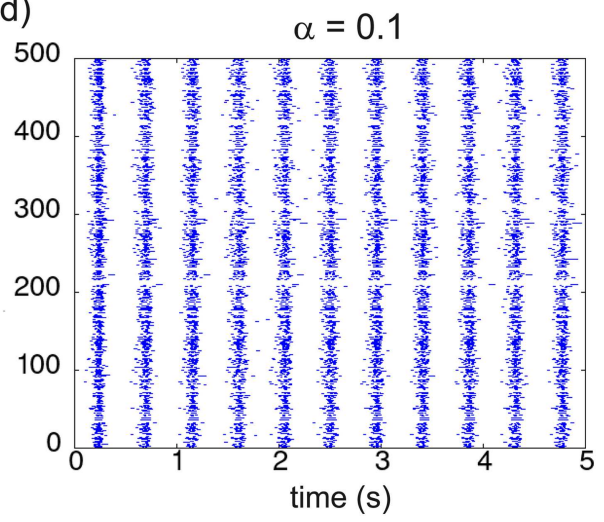

FIG. 3. Representatives raster plots of network activity for gradually higher connectivity probability $\alpha$. (a) Sporadic random activity. (b) Abundant random activity. (c) Beginning of coherent activity. (d) Strong coherent activity. The yellow band in (c) indicates the window of $50 \mathrm{~ms}$ for avalanche detection. 
although coherent activity is difficult to appraise. Synchronized activity shows up for $\alpha=0.05$ and strengthens as $\alpha$ grows.

The transition from erratic activity to full coherence as $\alpha$ increases resembles a (smooth) second order phase transition. To shed light on this transition, we quantify the size and duration of neuronal coordinated activations as a function of $\alpha$. For this analysis, we study the avalanches of activity as defined by Beggs and Plenz, ${ }^{26,27}$ in which an avalanche exists when at least two neurons activate together in a short time window. By analyzing the raster plots, we are able to detect the initiation of activity of any neuron, and then ascribe this and subsequent firings as an avalanche when the time-delay between neuronal coactivations is lower than $50 \mathrm{~ms}$.

We consider two major descriptors for avalanches, namely, "activation core" and "avalanche size." The activation core corresponds to the average number of neurons that have fired in every time bin within an avalanche, and portrays a characteristic size of coactive neurons. The activation core of an avalanche intuitively depicts the subset of strongly connected neurons. Indeed, we have numerically checked that the directed clustering coefficient of activation cores is (on average) three times larger than that of the underlying network. Avalanche size corresponds to the fraction of the neurons in the network that participated in a given avalanche.

Figure 4(a) shows the size of the avalanche core and avalanche duration as a function of $\alpha$. In general, the size of the avalanche core comprises about $2 \%-3 \%$ of the network for $\alpha \lesssim 0.01$ to gradually increase afterward. A value of $\alpha \simeq 0.02$ signals the onset of sufficient connectivity for large-scale neuronal coordination, in which the core of active neurons is 5\% of the network or higher. Avalanche duration shows a different trend. Although long lasting avalanches are observed for $\alpha \simeq 0.01$, they correspond to a large amount of small avalanches that overlap in time. Truly long lasting avalanches, i.e., chained activations of neurons, are observed for $\alpha \simeq 0.1$. Around this value and above, neuronal intercommunication is sufficiently strong to facilitate synchronous behavior and, therefore, the avalanche core can easily reach $25 \%$ of the network. This strong connectivity and intercommunication facilitates the formation of network-spanning activations. This is revealed by the avalanche size, as shown in Fig. 4(b), which reaches 1 for $\alpha \gtrsim 0.1$, i.e., the cascade of chained activations encompasses the entire network, in accordance to the raster plot of Fig. 3(d).

We note that the avalanche size and duration shown in Fig. 4(b) exhibit a rich variability for $\alpha$ in the range $0.01-0.1$. We understand this behavior in the context of the complex interplay between network structure and neuronal dynamics in spatial networks, as exposed by Orlandi et al. ${ }^{14}$ An avalanche may in principle start in several regions of the network, but its capacity to recruit more neurons and to grow depends on local and nonlocal network traits as well as activity-facilitation mechanisms such as noise amplification. Thus, a network at the edge of full connectivity with $\alpha \simeq 0.02$ may exhibit a rich variety of avalanche sizes and durations depending on the avalanche initiation point and propagation pattern.

From these analyses, we conclude that $\alpha=0.1$ is an adequate choice for the connectivity probability since it renders a dynamical regime of abundant coordinated activity that comprises the entire network. This regime also reflects well the characteristic activity of in vitro, living neuronal cultures grown on flat surfaces, ${ }^{14,15}$ in which the whole network fires coherently in a periodic manner as in Fig. 3(d).

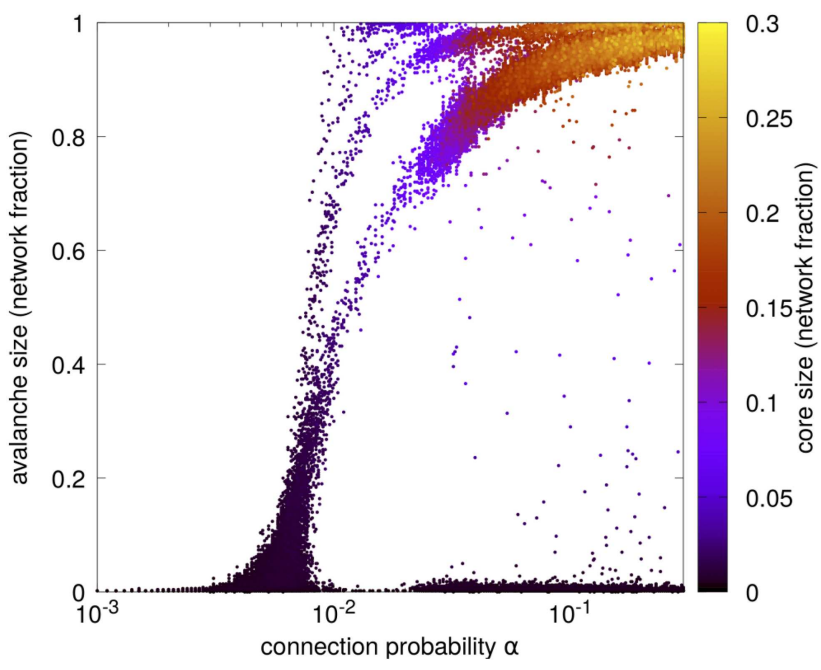

FIG. 4. Dependence of the avalanche size and avalanche core on the connection probability $\alpha$. Each point corresponds to an avalanche event, and $10^{4}$ events were analyzed.

Thus, we take $\alpha=0.1$ from here onward to generate our networks and deepen on the activity-connectivity relationship.

\section{B. Identification of central nodes}

Now, we turn our attention to different types of node centralities for the sake of analyzing the sensitivity of neuronal dynamics and network topology to the removal of particular nodes. Our final goal is to pinpoint the most damaging attack strategy. To this aim, we generated networks with $\alpha=0.1$ and explored which local topological measure most strongly facilitated the initiation of network activity. To quantify this facilitation, we introduced the "initiation probability" $p$, defined as the probability that a neuron belongs to the first group of strongly correlated neurons that trigger an avalanches event.

Orlandi et al. ${ }^{14}$ showed that the initiation of spontaneous activity in neuronal networks is grounded on two mechanisms, namely, (i) the amplification of noise by the circuitry and (ii) the convergence of activity toward basins of attraction where an avalanche finally initiates. In this context, those local network topological measures that promote signal propagation and whole-network communicability are the most attractive candidates to expose central nodes. Thus, of all possible topological measures, we concentrated our exploratory efforts in the four following ones:

- In-degree, ${ }^{19} k_{i}^{\text {in }}$ : number of links converging toward a particular node $i$. Biologically, it corresponds to the number of neurons whose axons connected with the dendritic tree of a particular neuron.

- Out-degree, ${ }^{19} k_{i}^{\text {out }}$ : number of links diverging from a particular node $i$. Biologically, it is the number of dendritic trees that the axon of a particular neuron connects to.

- Clustering coefficient, ${ }^{19} c c_{i}$ : defined as the probability that two neighbors of a particular node $i$ are also linked among themselves. 
(a)

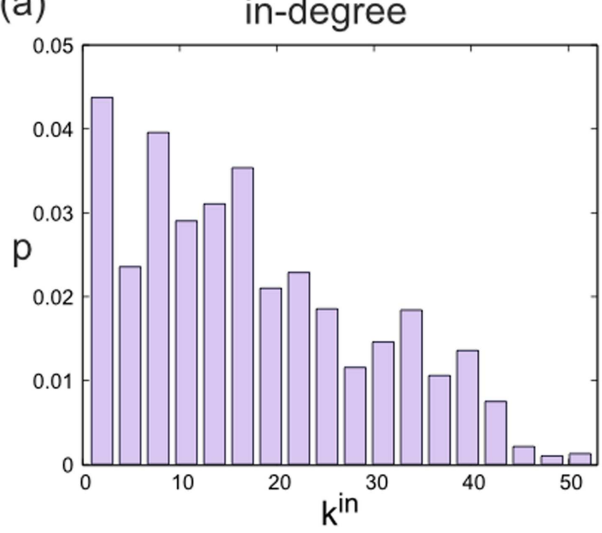

(c)

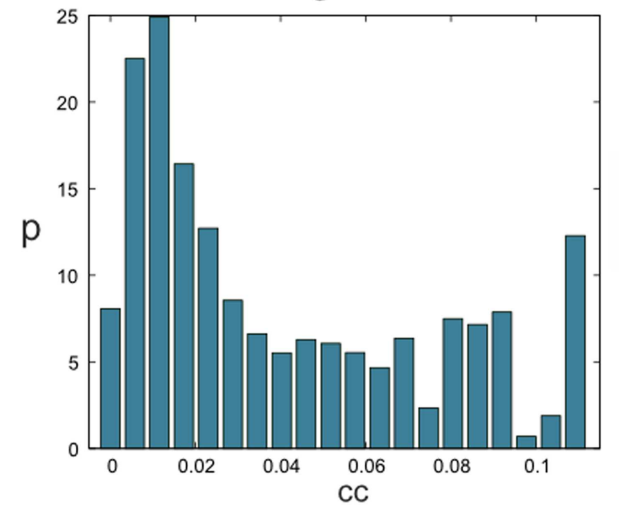

(b)

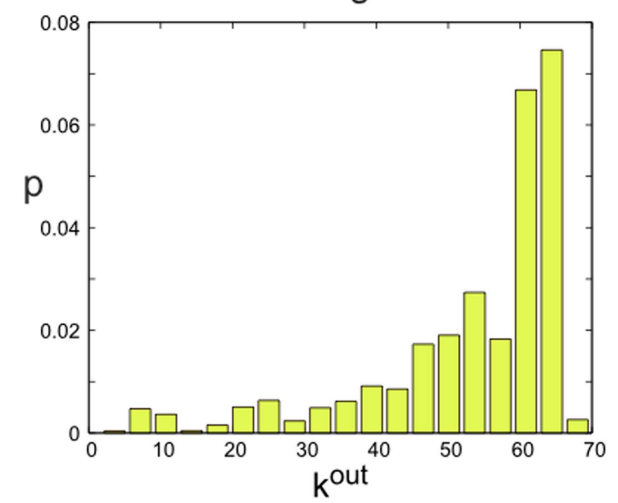

(d)

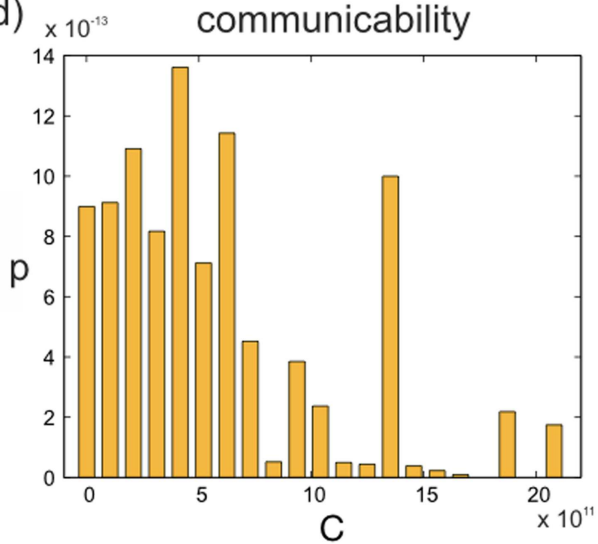

FIG. 5. Dependence of the avalanche initiation probability $p$ on 4 major topological measures. The out-degree $k_{\text {out }}$ is the measure that better portrays the sensitivity of a nodal property with dynamics.
Biologically, it quantifies the average probability to observe triangles of mutually connected neurons.

- Communicability, ${ }^{28} C_{i}$ : it accounts for all walks between node $i$ and all the nodes in the network (node $i$ included), weighting walks of length $h$ by a penalty factor of $1 / h$ !.

The analysis of these measures is shown in Fig. 5. The initiation probability decreases with the in-degree $k^{\text {in }}$ of nodes, their clustering coefficient $c c$, and their communicability $C$, while the out-degree $k^{\text {out }}$ exhibits the opposite trend.

A plausible explanation behind the increasing trend of $p$ with $k^{\text {out }}$ is that high out-degree nodes amplify activity with their neighborhood, thus boosting the probability to start an avalanche. In its turn, high $k^{\text {in }}$ nodes receive several inputs from potentially activated neighbors that are not necessarily transmitted to a large number of neighbors, thus restraining the activity of the network. This explanation is strengthened by the observation that, at a network level, a group of neurons shape the seed of an avalanche event when they can transmit pulses to as many neighboring neurons as possible.

Based on these results, we take the out-degree $k^{\text {out }}$ as the most relevant network property related to activity initiation. In the following, we investigate the implications that the gradual removal of the nodes with largest $k^{\text {out }}$ has on the collective dynamics of the cultures.

\section{Targeted attack and failure}

To assess the damage caused by the sequential deletion of nodes in decreasing order of $k^{\text {out }}$, we compare with the results obtained under random failures, i.e., when neurons are removed randomly. We disregarded depression mechanisms, i.e., we took $D_{i}=1 \forall i$ in Eq. (5). The rationale of this simplification is that we are primordially interested in the direct impact of central nodes removal on activity. The inclusion of depression would render memory effects, in which the removal of a neuron and subsequent dynamic alterations would be influenced by the past dynamical state of the network.

In Fig. 6(a), we evaluate the structural and dynamical consequences of targeted attack and random failure in our spatial networks. To this aim, we consider the "degree of activity" $A$, defined as the number of avalanches detected in $10 \mathrm{~s}$ and comprising at least $10 \%$ of the network. The $10 \%$ threshold is used to prevent small avalanches to dominate the statistics of activity. We then follow the evolution of $A$ as neurons in the network are removed, taking as control parameter the fraction of lost neurons in the network $q$. Network evolution is then plotted together with the giant component size $G$ as a function of $q$ to compare dynamical and structural disintegration. We note that, although these degradation diagrams correspond to $\alpha=0.1$, slightly larger (smaller) values of the connection probability $\alpha$ would shift the diagrams toward larger (smaller) values of $q$. 


\section{(a) degradation in biological spatial graphs}

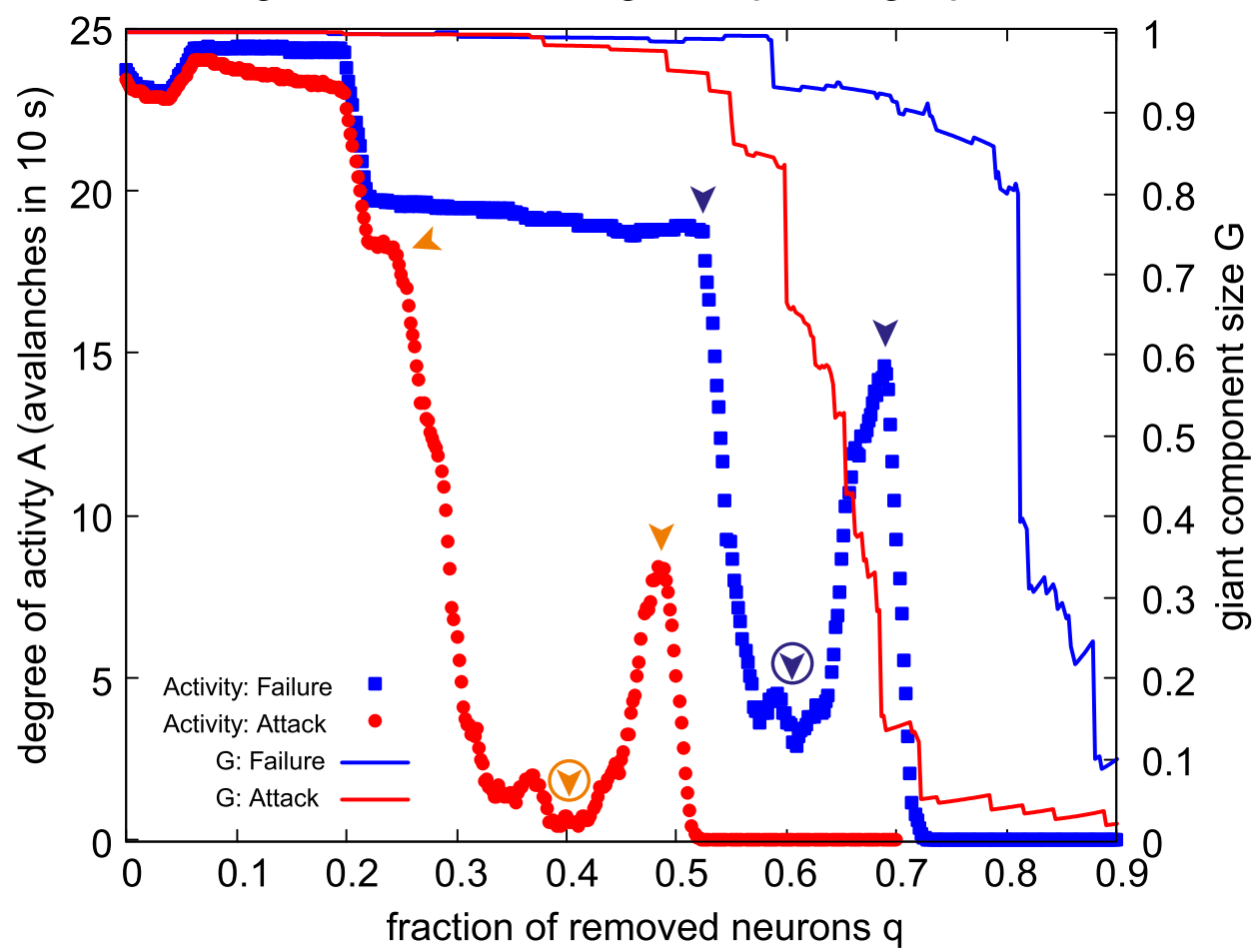

(b) initiation probability for failure

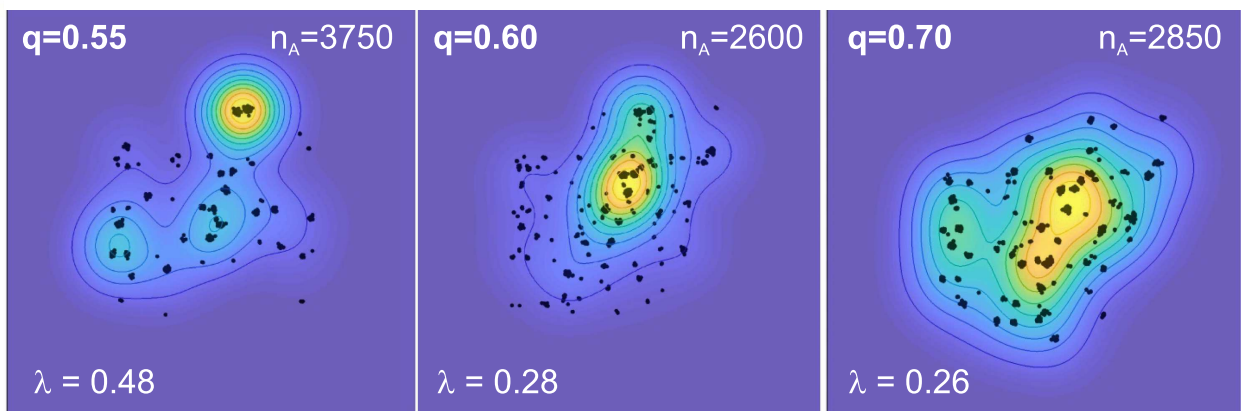

initiation probability for targeted attack min

$\max$
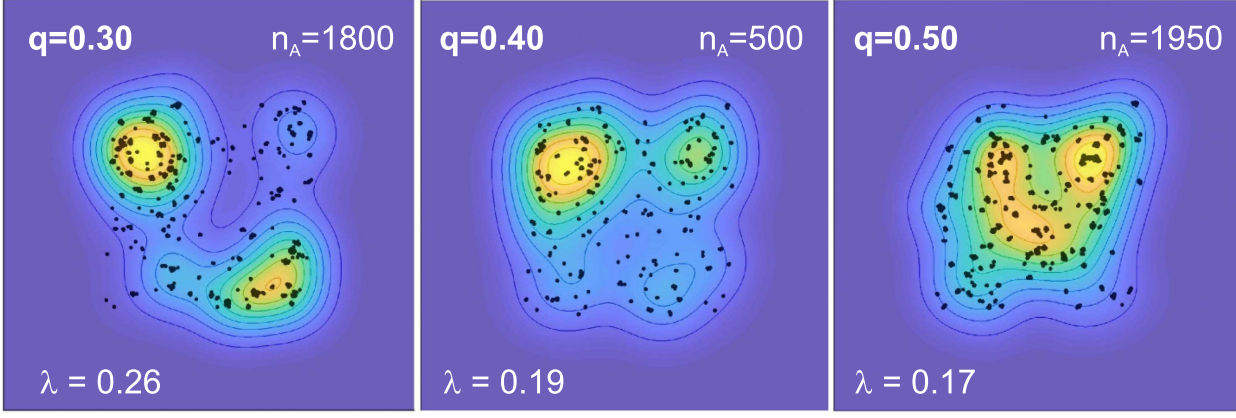

FIG. 6. Targeted attack and failure in spatial networks. (a) Dependence of network activity $A$ (symbols) and giant component size $G$ (lines) on the fraction of removed neurons $q$. Targeted attack (red) progressively deletes neurons with the highest $k_{\text {out }}$. Failure (blue) deletes arbitrary neurons. Arrowheads highlight points of interest along disintegration. (b) Probability distribution function of avalanche initiation points for key values of $q$ in a representative network realization. Avalanche data comprise all observed avalanches in $10 \mathrm{~min}$ of simulation. The brighter the color, the higher the probability for activity to initiate in a particular location. The number of avalanches used for statistics in each contour is indicated as $n_{A}$. Black dots show the location of each particular avalanche. $\lambda$ indicates the degree of aggregation of initiation points and is calculated as the gini coefficient of their spatial distribution. The higher the $\lambda$, the higher the aggregation. 
As a first result, we observe that dynamic alterations appear much earlier than structural ones. Indeed, the capacity of the network to fire and recruit activity has already decreased by $q \simeq 0.3-0.5$ in both damage scenarios, even though the giant component is still $G=1$.

As a second result, targeted attack clearly affects more strongly network activity than failure. Targeted attack just requires about $30 \%$ of network removal to begin compromising activity, while failure requires double damage, by $60 \%$. The result of removing large outdegree nodes in targeted attacks outlines their pivotal role in activity initiation. Indeed, as already exposed by Orlandi et al, ${ }^{14}$ a central mechanism governing the initiation of spontaneous activity is the amplification and propagation of activity throughout the network, a mechanism that is facilitated by out-degree nodes since neurons can send their pulses toward their neighborhood.

As a third result, the fall of network activity is not gradual but displays strong oscillations. The most prominent change occurs at $q \simeq 0.4$ for attack and $q \simeq 0.6$ for failure [Fig. 6(a), encircled arrowheads]. Here, almost silent networks suddenly "boost" in activity for a short range of $q$ until full collapse manifests. We hypothesize that the transition from quasisilence to activity raise is grounded on the change of a dynamic scenario from a network-spanning activity to a fragmented one. We denote by $\tilde{q}$ the critical fraction of removed neurons that separates the two scenarios. Thus, following our hypothesis, large-scale avalanches shape activity for $q<\tilde{q}$ and die out for $q \simeq \tilde{q}$; small avalanches then emerge in local yet abundant regions for $q>\tilde{q}$.

To verify this hypothesis, we carried out additional analyses. In a first analysis [Fig. 6(b)], we considered a representative network realization and studied the distribution of initiation points, i.e., the location in the $x-y$ space of a particular avalanche. In the panels, each black dot corresponds to the focus of avalanche initiation. For weak damage (left panels of the figure), the avalanches' probability distribution function show peaks at specific areas, which appear as bright yellow in the panels. This compactness in the initiation probability is a trait of network-spanning avalanches in spatially-embedded networks. ${ }^{14}$ The strong concentration of initiation points in a single spot is quantified through the gini coefficient $\lambda$. A value of $\lambda=0$ would correspond to an ensemble of homogeneously distributed initiation points, while $\lambda=1$ would correspond to the concentration of all points in a single spot. The relative large value of $\lambda$ indicates that concentration is indeed strong. However, $\lambda$ drops by $35 \%$ at $q \simeq \tilde{q}$ (central panels) and keeps decreasing as damage accentuates (right panels). Thus, the distribution of initiation points is markedly sparse for $q \gtrsim \tilde{q}$, with no distinctive initiation areas, and reveals a spatially fragmented dynamics.

In a second analysis (Fig. 7), we computed the distribution of avalanche sizes at the vicinity of $\tilde{q}$. We compared the avalanche distributions at two $q$ values, termed $q_{A}$ and $q_{B}$ (Fig. 7 , insets). The former (a)

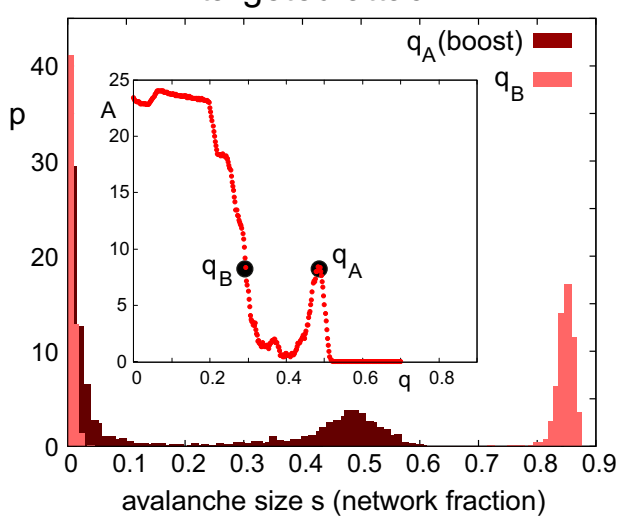

(c)

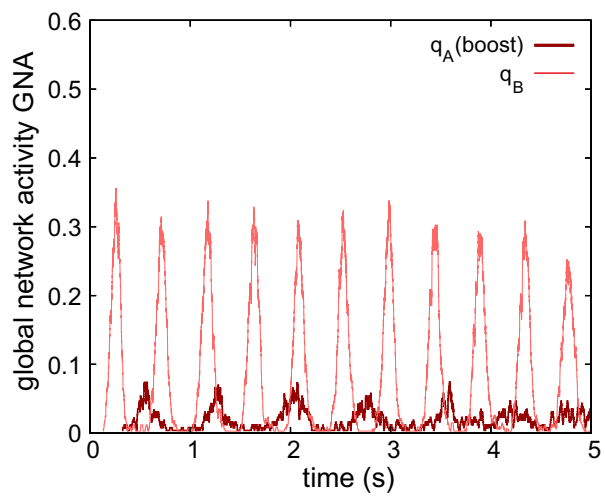

(b) failure

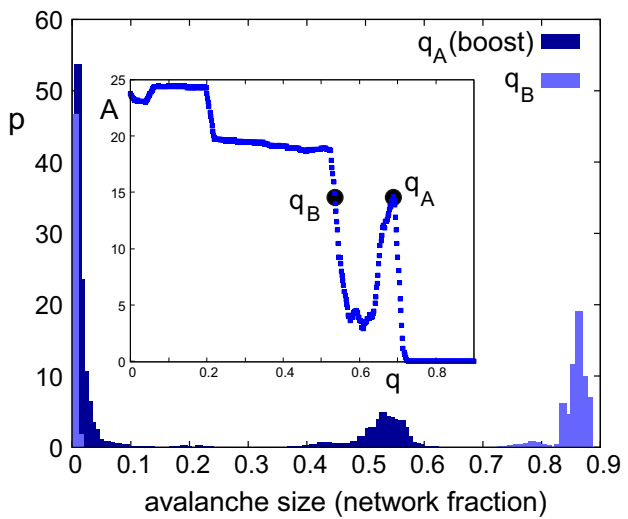

(d)

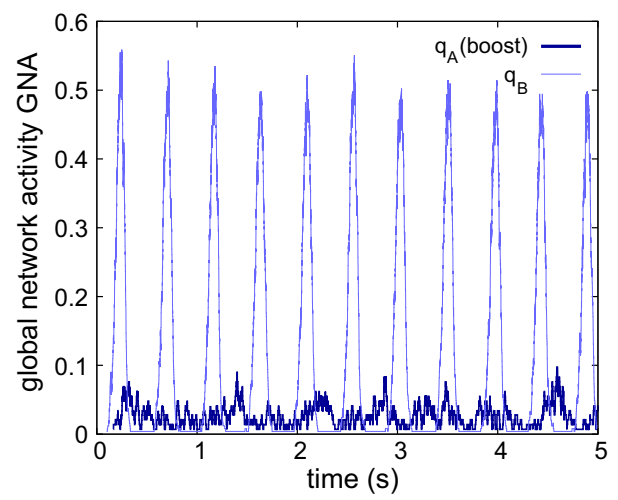

FIG. 7. Fragmentation of activity in spatial networks upon damage. (a) Avalanche distributions for targeted attack at two $q$ values, a first one corresponding to the peak of boosted activity and that renders segregated activity and a second one corresponding to a weaker damage equivalent that renders whole-network activations. Note that the avalanche size $s$ is measured as the fraction of neurons involved relative to the size of the remaining network at the corresponding $q$. (b) Equivalent analysis for failure. In both (a) and (b), the insets portray the $A(q)$ curves for clarity. (c) Comparison of the degree of global spontaneous activity for the two $q$ values under targeted attack, illustrating the change from a network-spanning activity to a fragmented one. (d) Equivalent analysis for failure. 
corresponds to the peak of "boosted" activity, and the latter corresponds to the first $q<\tilde{q}$ value that renders the same activity. For both targeted attack and failure [Figs. 7(a) and 7(b)], avalanche sizes at moderate damage $q_{B}$ encompass either very small events of size $s \lesssim 0.05$ (measured as network fraction) or quasi-whole-network activations of $s \simeq 0.85$. No avalanches are observed in between these extrema, reflecting the existence of sufficient network cohesion for whole-network communication. By contrast, avalanche sizes at strong damage $q_{A}$ substantially shift toward lower values. The biggest avalanches encompass $s \simeq 0.6$, but a wide spectrum of avalanche sizes exist for $s \lesssim 0.6$. Thus, these results show that strong damage breaks the network apart into two or more dynamically isolated regions. Further suppression of nodes gradually shrinks these regions even further until no activity is possible.

To illustrate the contrasting dynamics between $q_{A}$ and $q_{B}$, Figs. 7 (c) and 7(d) show the evolution of activity for a representative network, comparing both damage scenarios and $q$ values. In the plots, global network activity (GNA) is the fraction of neurons in the network that fire together in a time bin of $50 \mathrm{~ms}$ and provides a quick overview of the typical avalanches along the simulation. Qualitatively, the transition from moderate damage at $q_{B}$ to strong damage at $q_{A}$ drops network coactivations in both targeted attack and failure, with practically no coactivations bigger than $10 \%$ of the network.

\section{Effects of spatial embedding}

Finally, we investigate the evolution of network activity $A$ as a function of $q$ in equivalent Erdös-Rényi (ER) random networks to gain further insight into the importance of spatial embedding in damage (Fig. 8). The ER networks are constructed preserving the same number of nodes $N$ and mean degree $\langle k\rangle$ as our synthetic neuronal cultures (see inset in Fig. 2). As expected, no significant differences show up between targeted attacks and random failures since the degree heterogeneity of ER is small. In addition, the ER topology effectually shapes long range connections among neurons that strengthen the giant component, which starts decaying at much higher values of $q$ than in the spatial construction. This cohesiveness indicates that network-wide communication among neurons is always maintained. By comparing the spatial network subjected to random failures with the behavior of the ER graph, we observed that for $q \lesssim 0.5$, the activity of the latter is lower than that in spatial networks. However, the activity loss at $q>0.5$ occurs in a more gradually manner. Indeed, no fluctuations or boosted activity are observed at high $q$ values since activity in the ER networks always encompasses the whole system up to full collapse, i.e., the characteristic fragmentation of activity observed in the spatial construction is not present here.

To better understand the origin of the sudden boost of activity in spatial networks, we now investigate the "effective connectivity" of the networks before the boost and at the peak of it. The effective connectivity helps identifying characteristic patterns of coordinated activity among neurons and therefore allows to pinpoint spatiotemporal alterations in network dynamics.

Effective connectivity was analyzed as follows. We first considered the raster plots of spontaneous activity corresponding to about $10 \mathrm{~min}$ of activity, and resampled the data in $50 \mathrm{~ms}$ bins to consider time series of tractable size. We then used Transfer Entropy (TE)

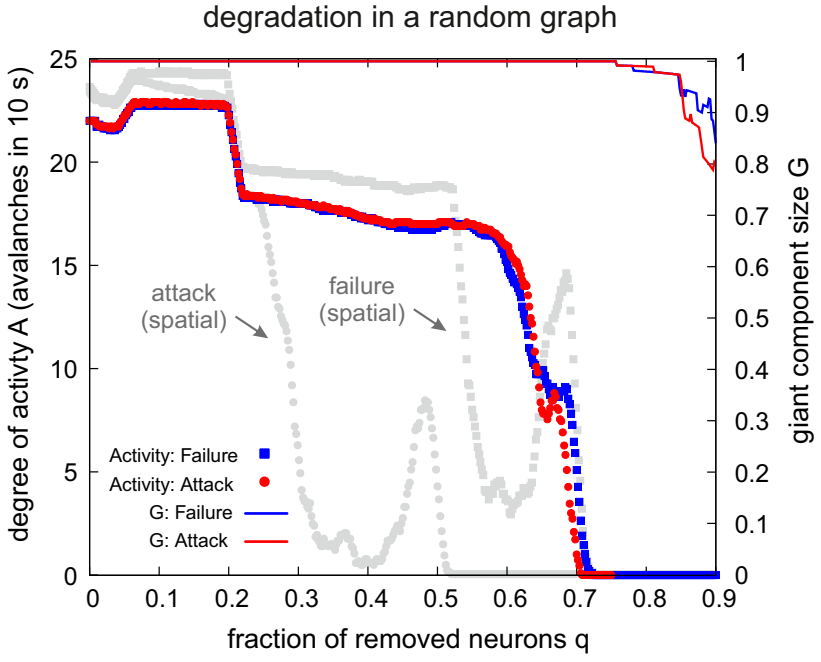

FIG. 8. Targeted attack and failure in Erdös-Rényi (ER) random graphs. Both attack scenarios follow a similar trend with small quantitative differences. The equivalent disintegrations for spatial networks are shown in gray for an easier comparison of the action of damage in the two network schemes.

as in Refs. 29 and 18 to determine causal relationships among firing neurons. An effective connection between neurons $i$ and $j$ was deemed significant whenever their $\mathrm{TE}_{i j}$ score exceeded the mean +2 standard deviations of the joint distribution. Each inferred effective network was directed and weighted. We observed that typically a subset of 100 neurons contained the strongest links. We, therefore, used this subset as a representation of the network, thresholded the adjacency matrix values to $\{0,1\}$ and analyzed its statistical properties. Specifically, we investigated the existence of communities using a fast implementation of the Louvain's algorithm on the largest connected component. ${ }^{30}$ In our case, communities signal groups of neurons that tend to fire independently within microcircuits. We quantified the degree of fragmentation through the "community statistic" $M$, which was calculated using the Brain Connectivity Toolbox. ${ }^{31} M$ intuitively portrays the degree of fragmentation of the network into communities. A value $M=0$ indicates no communities, and gradually larger $M$ values indicate a higher segregation.

The results are shown in Fig. 9(a). For both failure and attack, $q$ values before the boost show a strongly connected network from a dynamical perspective $(M=0.07)$, indicating that all neurons participate in the same avalanches. However, at the $q$ values of the boost, both actions lead to a dynamically segregated network. For failure, two communities are observed $(M=0.41)$, pointing out that two groups of neurons are firing with different dynamical structures. For attack, a larger $M=0.60$ is measured, indicating a stronger fragmentation that shapes four communities. The high number of communities during attack strengthens the message that this action is much more aggressive in destroying the dynamical cohesiveness of the network than failure. Notably, ER graphs [Fig. 9(a), right panels] do not exhibit this fragmentation of the dynamics into communities, with the networks maintaining a coherent activity until it ceases. 
(a) Failure Spatial

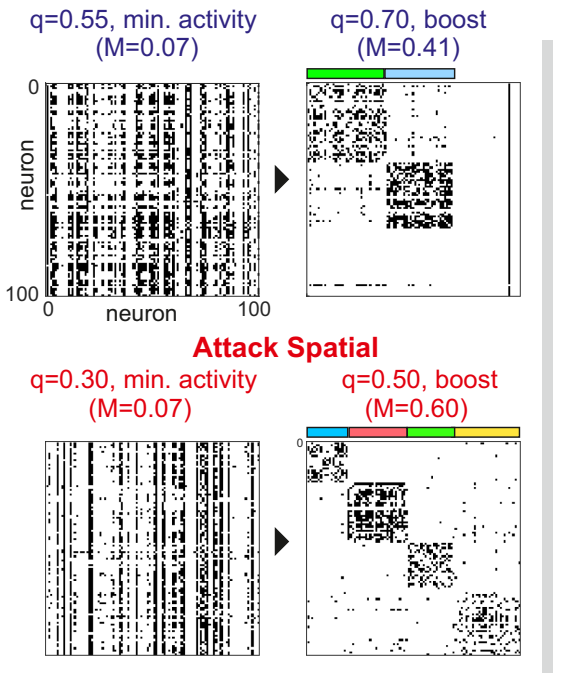

Failure ER

$q=0.66$

$(\mathrm{M}=0.13)$

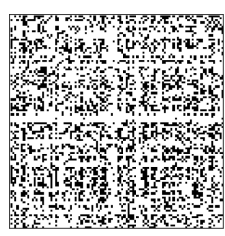

Attack ER

$q=0.67$

$(\mathrm{M}=0.18)$

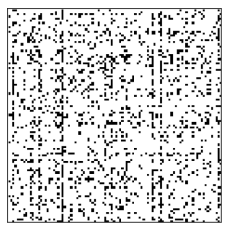

(b)

Spatial segregation of activity at 'boost' failure $(q=0.70)$
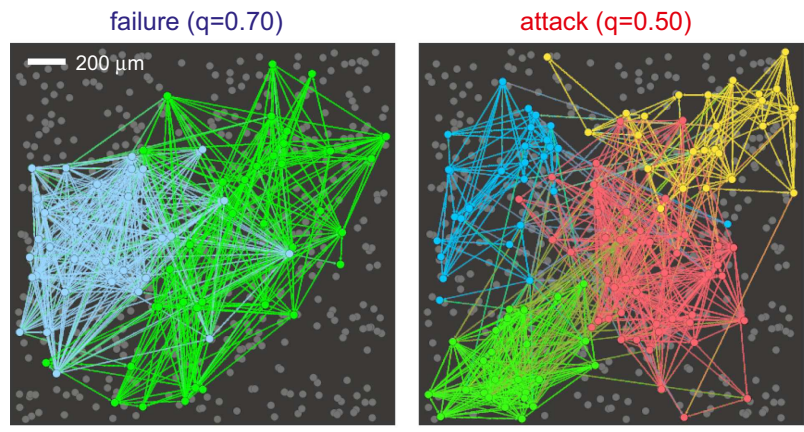

FIG. 9. Effective connectivity in spatial and ER graphs. (a) Thresholded adjacency matrices of the 100 neurons with top-ranked effective links (black dots). Data correspond to the "preboost" and "boost" $q$ points of Fig. 6. The community statistic $M$ indicates the tendency of the effective network to segregate into communities. The higher $M$, the higher the segregation. Spatial networks exhibit segregation during the episodes of boosted activity. Both the number of communities and the degree of segregation are higher in the attack scenario. ER graphs do not segregate upon damage and remain activating as a global system. The color bars above the adjacency matrices label each community for easier identification in the below spatial maps. (b) Spatial distribution of the inferred communities, portraying those groups of neurons that distinctively coactivate together, shaping dynamical microcircuits that are practically independent. Circles are neurons and arrows are effective links.

To demonstrate that the dynamical fragmentation in spatial networks was also physical, i.e., that it involved the concentration of activity into specific areas of the network, we analyzed the spatial location of the inferred communities. As shown in Fig. 9(b), the above inferred communities correspond to compact, distinct regions of activity in the network with weak overlap between themselves, thus shaping clear spatial subnetworks with rich dynamics.

The effective connectivity analysis, together with the comparison between spatial and ER graph, supports the message that spatial embedding facilitates local connectivity among neurons, which translates into: (i) a balance between local dynamic amplification and large-scale communication that fosters whole-network activity and (ii) the preservation of sufficiently connected local circuits that, even for strong damage, are able to maintain high levels of activity.

\section{DISCUSSION AND CONCLUSIONS}

The analysis of damage in neuronal circuits is important to understand their resilience to the loss of neurons or connections. The study presented here is an effort to model the impact of spatial embedding-an intrinsic feature of biologically neuronal circuits-on the degradation of neuronal activity as neurons are removed. The important differences in the degradation process between ER and spatial networks already advocate for paying closer attention to spatially-inherited features and their relevance in the structure-dynamics relationship.

Our work illustrates the complex interplay between structural circuitry and intrinsic neuronal dynamics. The neuronal circuitry shapes the backbone for the transfer of information between neurons and delineates the communication flows. However, dynamics may be even more important as shown by the large values of the giant component when the network was already at the verge of silencing. Thus, topological properties such as degree, hubs, or small-worldness are important, but they may be irrelevant if the network cannot fire. Indeed, the combination of both structure and dynamics, together with noise, orchestrates spontaneous activity, which is a fundamental feature of all living neuronal circuits and whose alteration may cause substantial deficits, particularly in the brain.

Targeted attack and failure were very different, with the former compromising activity for a much lower fraction of deleted nodes. We used targeted attack based on out-degree nodes removal. This choice is in agreement with other studies. ${ }^{3,7,10}$ We note, however, that given the complex coupling between structure and dynamics, one could define multiattack features by combining topological damage with dynamical one. The latter could be designed as the deletion of activity initiation points. We hypothesize that such an action could substantially deplete activity, since both the amplification of activity and its convergence toward specific areas would be obstructed.

The exploration of spatial features and their impact in structure and dynamics can go beyond the analysis shown here. As a first consideration, we used in the simulations a homogeneous distribution of neurons, but living neuronal networks often exhibit strong neuronal aggregation that shapes locality strongly coupled communities. ${ }^{33,34}$ As a second consideration, we used a relatively short axonal length in all neurons based on biological observations in neuronal cultures. Axonal lengths, however, can potentially be very different in the same system, thereby affecting the balance between short and long range connections.

In this direction, Hernández-Navarro et al. ${ }^{17}$ investigated the importance of these two spatial features-aggregation and axonal length-on network response and observed that the metric-inherited correlations could shape very different dynamic regimes and even rule out details such as the distribution of connections. For instance, they showed that spatial embedding became irrelevant when the 
average axonal length was on the order of the system size. Translated to our simulations, these results indicate that the response to damage could vary depending on neuronal positions and axonal lengths. We observed that much longer axonal lengths would shape damage scenarios closer to ER. The perspectives that our analysis opens are, therefore, very enlightening. We could, for instance, investigate damage in different stages of growing axons and pinpoint the impact of damage during development.

An important strength of our work is that it lays the foundation for mimicking the structure of living circuits, in particular, neuronal cultures, and study their behavior upon damage. Recent studies $^{35}$ have shown the potential of neuroengineering to prepare specific configurations such as modular circuits. Thus, we can use our approach to predict the vulnerability of certain designs and explore possible recovery mechanisms of the living networks.

The spatial networks are more resilient to failure than their equivalent random graphs. The reason is that, for the spatial construction, the network breaks apart into localized microcircuits that maintain rich activity, which does not occur in ER networks. Thus, spatial embedding enhances resilience at the price of losing long range communication. In the context of highly modular architectures, this long range loss turns into a favorable mechanism that allows the maintenance of activity in localized regions. We also note that, if damage is viewed as a controlled silencing of neurons, spatial features provide direct mechanisms to dynamically couple microcircuits, allowing to tune the dynamical state of the system from whole-network activation to segregated activity, in the same fashion as observed in the functional organization of engineered neuronal cultures $^{35}$ and brain networks. ${ }^{36}$

In a nutshell, our work sheds light on two central results. First, the dynamical patterns are as important as the structural ones for understanding the effects of damage and the resilience of complex networks. Thus, dynamics should not be disregarded. Second, although spatial networks seem less resilient, they have an important advantage, as they facilitate activity to be retained in localized circuits. This property shapes an intrinsic mechanism for resilience that may be pivotal for survival of biological networks.

\section{ACKNOWLEDGMENTS}

We acknowledge financial support from the Spanish Ministerio de Economia y Competitividad through Project Nos. FIS2015-71582-C2, FIS2016-78507-C2-2-P, FIS2017-87519-P, and FIS2017-90782-REDT (IBERSINC), from the Generalitat de Catalunya through Grant No. 2017-SGR-1061, and from the Departamento de Industria e Innovación del Gobierno de Aragón y Fondo Social Europeo (FENOL group). S.F.L. acknowledges financial support from Gobierno de Aragon through a doctoral fellowship.

\section{REFERENCES}

${ }^{1}$ A. Fornito, A. Zalesky, and M. Breakspear, "The connectomics of brain disorders," Nat. Rev. Neurosci. 16, 159-72 (2015).

${ }^{2}$ C. Gratton, E. M. Nomura, F. Perez, and M. D. Esposito, "Focal brain lesions to critical locations cause widespread disruption of the modular organization of the brain,” J. Cogn. Neurosci. 24(6), 1275-1285 (2012).

${ }^{3}$ J. Alstott, M. Breakspear, P. Hagmann, L. Cammoun, and O. Sporns, "Modeling the impact of lesions in the human brain," PLoS Comput. Biol. 5(6), 1-12 (2009).
${ }^{4}$ R. L. Buckner, J. Sepulcre, T. Talukdar, F. M. Krienen, H. Liu, T. Hedden, J. R. Andrews-Hanna, R. A. Sperling, and K. A. Johnson, "Cortical hubs revealed by intrinsic functional connectivity: Mapping, assessment of stability, and relation to Alzheimer's disease," J. Neurosci. 29(6), 1860-1873 (2009).

${ }^{5}$ E. J. Sanz-Arigita, M. M. Schoonheim, J. S. Damoiseaux, S. A. R. B. Rombouts, E. Maris, F. Barkhof, P. Scheltens, and C. J. Stam, "Loss of "small-world" networks in Alzheimer's disease: Graph analysis of FMRI resting-state functional connectivity," PLoS One 5(11), 1-14 (2010).

${ }^{6}$ L.-D. Lord, A. B. Stevner, G. Deco, and M. L. Kringelbach, Understanding principles of integration and segregation using whole-brain computational connectomics: Implications for neuropsychiatric disorders," Philos. Trans. R. Soc. A 375(2096), 20160283 (2017).

${ }^{7}$ M. Kaiser, R. Martin, P. Andras, and M. P. Young, "Simulation of robustness against lesions of cortical networks," Eur. J. Neurosci. 25(10), 3185-3192 (2007).

${ }^{8}$ K. E. Joyce, S. Hayasaka, and P. J. Laurienti, "The human functional brain network demonstrates structural and dynamical resilience to targeted attack," PLoS Comput. Biol. 9(1), 1-11 (2013).

${ }^{9}$ J. Gómez-Gardeñes, P. Echenique, and Y. Moreno, "Immunization of real complex communication networks," Eur. Phys. J. B 49(2), 259 (2006).

${ }^{10}$ M. Rubinov, A. R. McIntosh, M. J. Valenzuela, and M. Breakspear, "Simulation of neuronal death and network recovery in a computational model of distributed cortical activity," Am. J. Geriatr. Psychiatry 17(3), 210-217 (2009).

${ }^{11}$ S. Feldt, P. Bonifazi, and R. Cossart, "Dissecting functional connectivity of neuronal microcircuits: Experimental and theoretical insights," Trends. Neurosci. 34(5), 225-236 (2011).

${ }^{12}$ D. Holstein, A. V. Goltsev, and J. F. F. Mendes, "Impact of noise and damage on collective dynamics of scale-free neuronal networks," Phys. Rev. E 87, 032717 (2013).

${ }^{13}$ L. J. Millet and M. U. Gillette, "Over a century of neuron culture: From the hanging drop to microfluidic devices,” Yale J. Biol. Med. 85(4), 501 (2012).

${ }^{14} \mathrm{~J}$. G. Orlandi, J. Soriano, E. Alvarez-Lacalle, S. Teller, and J. Casademunt, "Noise focusing and the emergence of coherent activity in neuronal cultures," Nat. Phys. 9(9), 582 (2013).

${ }^{15} \mathrm{E}$. Tibau, M. Valencia, and J. Soriano, "Identification of neuronal network properties from the spectral analysis of calcium imaging signals in neuronal cultures," Front. Neural Circuit. 7, 199 (2013).

${ }^{16} \mathrm{~S}$. Okujeni, S. Kandler, and U. Egert, "Mesoscale architecture shapes initiation and richness of spontaneous network activity," J. Neurosci. 37(14), 3972-3987 (2017).

${ }^{17}$ L. Hernández-Navarro, J. G. Orlandi, B. Cerruti, E. Vives, and J. Soriano, "Dominance of metric correlations in two-dimensional neuronal cultures described through a random field Ising model," Phys. Rev. Lett. 118, 208101 (2017).

${ }^{18}$ E. Tibau, A. Ludl, S. Rudiger, J. G. Orlandi, and J. Soriano, "Neuronal spatial arrangement shapes effective connectivity traits of in vitro cortical networks," IEEE Trans. Network Sci. Eng. (2018).

${ }^{19}$ M. E. J. Newman, Networks: An Introduction (Oxford University Press, 2010).

${ }^{20}$ C. Schmeltzer, J. Soriano, I. M. Sokolov, and S. Rüdiger, "Percolation of spatially constrained Erdös-Rényi networks with degree correlations," Phys. Rev. E 89, 012116 (2014).

${ }^{21}$ E. M. Izhikevich, "Simple model of spiking neurons," IEEE Trans. Neural Networks 14(6), 1569-1572 (2003).

${ }^{22}$ E. Alvarez-Lacalle and E. Moses, "Slow and fast pulses in 1-D cultures of excitatory neurons," J. Comput. Neurosci. 26(3), 475-493 (2009).

${ }^{23} \mathrm{M}$. Tsodyks and H. Markram, "The neural code between neocortical pyramidal neurons depends on neurotransmitter release probability," Proc. Natl Acad. Sci. 94(2), 719-723 (1997).

${ }^{24}$ M. Tsodyks, A. Uziel, H. Markram et al., "Synchrony generation in recurrent networks with frequency-dependent synapses," J. Neurosci. 20(1), 825-835 (2000).

${ }^{25} \mathrm{D}$. Golomb and Y. Amitai, "Propagating neuronal discharges in neocortical slices: Computational and experimental study," J. Neurophysiol. 78(3), 1199-1211 (1997).

${ }^{26}$ J. M. Beggs and D. Plenz, "Neuronal avalanches in neocortical circuits," J. Neurosci. 23(35), 11167-11177 (2003).

${ }^{27}$ J. M. Beggs and D. Plenz, "Neuronal avalanches are diverse and precise activity patterns that are stable for many hours in cortical slice cultures," J. Neurosci. 24(22), 5216-5229 (2004). 
${ }^{28}$ E. Estrada and N. Hatano, "Communicability in complex networks," Phys. Rev. E 77(3), 036111 (2008).

${ }^{29}$ O. Stetter, D. Battaglia, J. Soriano, and T. Geisel, "Model-free reconstruction of excitatory neuronal connectivity from calcium imaging signals," PLoS Comput. Biol. 8(8), 1-25 (2012).

${ }^{30}$ Y. Sun, B. Danila, K. Josić, and K. E. Bassler, "Improved community structure detection using a modified fine-tuning strategy," Europhys. Lett. 86(2), 28004 (2009).

${ }^{31}$ M. Rubinov and O. Sporns, "Complex network measures of brain connectivity: Uses and interpretations," NeuroImage 52(3), 1059-1069 (2010).

${ }^{32}$ A. G. Blankenship and M. B. Feller, "Mechanisms underlying spontaneous patterned activity in developing neural circuits," Nat. Rev. Neurosci. 11(1), 18 (2010).

${ }^{33}$ D. de Santos-Sierra, I. Sendiña-Nadal, I. Leyva, J. A. Almendral, S. Anava, A. Ayali, D. Papo, and S. Boccaletti, "Emergence of small-world anatomical networks in self-organizing clustered neuronal cultures," PLoS One 9(1), 1-8 (2014).

${ }^{34}$ S. Teller, C. Granell, M. De Domenico, J. Soriano, S. Gómez, and A. Arenas, "Emergence of assortative mixing between clusters of cultured neurons," PLoS Comput. Biol. 10(9), 1-17 (2014).

${ }^{35} \mathrm{H}$. Yamamoto, S. Moriya, K. Ide, T. Hayakawa, H. Akima, S. Sato, S. Kubota, T. Tanii, M. Niwano, S. Teller, J. Soriano, and A. Hirano-Iwata, "Impact of modular organization on dynamical richness in cortical networks," Sci. Adv. 4(11), eaau4914 (2018).

${ }^{36} J$. Gómez-Gardeñes, G. Zamora-López, Y. Moreno, and A. Arenas, "From modular to centralized organization of synchronization in functional areas of the cat cerebral cortex," PLoS One 5(8), e12313 (2010).

${ }^{37}$ G. Zamora-López, C. Zhou, and J. Kurths, "Cortical hubs form a module for multisensory integration on top of the hierarchy of cortical networks," Front. Neuroinform. 4, 1 (2010). 